\title{
Black hole motion in Euclidean space as a diffusion process II
}

\author{
K. Ropotenko \\ State Service for Special Communication and \\ Information Protection of Ukraine, \\ 13 Solomianska str., Kyiv, 03680, Ukraine \\ ropotenko@ukr.net
}

\begin{abstract}
A diffusion equation approach to black hole thermodynamics in Euclidean sector is proposed. A diffusion equation for a generic KerrNewman black hole in Euclidean sector is derived from the Bloch equation. Black hole thermodynamics is also derived and it is found, in particular, that the entropy of a generic Kerr-Newman black hole is the same, apart from the logarithmic corrections, as the BekensteinHawking entropy of the black hole.
\end{abstract}

In [1], I derived a diffusion equation for a Schwarzschild black hole from the Bunster-Carlip equations and showed that the black hole evolution in Euclidean sector exhibits a diffusion process. Namely I showed that the Bunster-Carlip equations

$$
\begin{gathered}
\frac{\hbar}{i} \frac{\partial \psi}{\partial t}+M \psi=0, \\
\frac{\hbar}{i} \frac{\partial \psi}{\partial \Theta}-\frac{A}{8 \pi G} \psi=0,
\end{gathered}
$$

where $t$ is the lapse of asymptotic proper time at spatial infinity and $\Theta$ is the lapse of the hyperbolic angle at the horizon, transform to the equation

$$
\frac{\partial \rho}{\partial \Theta_{\mathrm{E}}}=D \frac{\partial^{2} \rho}{\partial t_{\mathrm{E}}^{2}}
$$


for the probability density $\rho=\left|\psi\left(t_{\mathrm{E}}, \Theta_{\mathrm{E}}\right)\right|^{2}$ under Euclidean continuation $\Theta_{\mathrm{E}}=i \Theta$ and $t_{\mathrm{E}}=i t$. In general, the Euclidean time coordinate $t_{\mathrm{E}}$ behaves like a spatial coordinate. But (3) is an one-dimensional diffusion equation in the temporal $\Theta_{\mathrm{E}}$ and spatial $t_{\mathrm{E}}$ coordinates with the diffusion coefficient $D=2 G \hbar$. So I reinterpreted the Euclidean time coordinate $t_{\mathrm{E}}$ as a spatial coordinate and $\Theta_{\mathrm{E}}$ as a temporal coordinate. In place of $\Theta_{\mathrm{E}}$ and $t_{\mathrm{E}} \mathrm{I}$ will hereafter write $\Theta$ and $\tau$. After analytical continuation to the cyclic imaginary time, we deal with a quantum system at a finite temperature, so the black hole should be described not by the probability density $\rho(x)$ but by the canonical density matrix $\rho\left(x, x^{\prime} ; \beta\right)$. Determining $\rho\left(x, x^{\prime} ; \beta\right)$ I found that the entropy of a Schwarzschild black hole is the same, apart from the logarithmic corrections, as the Bekenstein-Hawking entropy.

In the literature, the path integral approach is the only approach to black hole thermodynamics in Euclidean quantum gravity. In this note I propose an alternative diffusion equation approach. I derive a diffusion equation for the density matrix of a generic Kerr-Newman black hole in Euclidean sector immediately from the Bloch equation. I also derive black hole thermodynamics and find, in particular, that the entropy of a Kerr-Newman black hole is the same, apart from the logarithmic corrections, as the Bekenstein-Hawking entropy of the black hole.

I begin with the Bloch equation [2]

$$
\frac{\partial \rho}{\partial \beta}=-H \rho
$$

for a Schwarzschild black hole at a temperature $T_{\mathrm{H}}=\beta^{-1}$. The (unnormalized) canonical density matrix has the form $\rho(\beta)=e^{-\beta H}$. Note that here, as in ordinary statistics, the form of the density matrix must be regarded only as a postulate, to be justified solely on the basis of agreement of its predictions with the thermodynamical properties of black holes. As before, I adopt the Euclidean approach and consider the black hole motion in Euclidean sector in the temporal $\Theta$ and spatial $\tau$ coordinates, so that $\rho(\beta)=\rho\left(\tau, \tau^{\prime} ; \beta\right)$ in coordinate representation. For physical applications $\Theta$ should be set equal to $2 \pi$, so that $\beta=2 \pi / k$, where $k$ is the surface gravity. But for the purposes of thermodynamic analysis I shall keep $\Theta$ arbitrary and put $\Theta=2 \pi$ only in some final results. Therefore, (4) reads

$$
\frac{\partial \rho}{\partial \Theta}=-\frac{1}{k} H \rho
$$


Next, I define the Hamiltonian of a black hole with the mass $M$ as that of a free particle moving along the coordinate $\tau$

$$
H=\frac{p^{2}}{2 M},
$$

and obtain

$$
\frac{\partial \rho}{\partial \Theta}=D \frac{\partial^{2} \rho}{\partial \tau^{2}}
$$

where $D=\hbar / 2 k M$. This is an one-dimensional diffusion equation, and we can write down its solution readily:

$$
\rho\left(\tau, \tau^{\prime} ; \Theta\right)=\frac{1}{\sqrt{4 \pi D \Theta}} \exp \left[-\frac{\left(\tau-\tau^{\prime}\right)^{2}}{4 D \Theta}\right],
$$

where the proportionality factor is chosen such that

$$
\rho\left(\tau, \tau^{\prime} ; 0\right)=\delta\left(\tau-\tau^{\prime}\right) .
$$

The crucial property of the black hole solutions in Euclidean sector is their periodicity in the imaginary time, $\tau \sim \tau+\beta$, where $\beta=2 \pi / k$. Therefore

$$
\rho(\tau, \tau ; \Theta)=\frac{1}{\sqrt{4 \pi D \Theta_{\mathrm{E}}}} \exp \left(-\frac{\beta^{2}}{4 D \Theta}\right) .
$$

For a linear system of length $\beta=2 \pi / k$, the integration over $\tau$ gives

$$
Z(\beta)=\int_{0}^{\beta} \rho(\tau, \tau) d \tau=\frac{\beta}{\sqrt{4 \pi D \Theta}} \exp \left(-\frac{\beta^{2}}{4 D \Theta}\right) .
$$

This is the partition function for a Schwarzschild black hole. Differentiating with respect to the $\beta$ and then putting $\Theta=2 \pi$, we obtain the internal energy

$$
E=-\frac{\partial \ln Z(\beta)}{\partial \beta}=M-\beta^{-1}
$$

It is the same, apart from the Hawking temperature, as the mass of the black hole. The entropy of the black hole is given by

$$
S=\ln Z+\beta E=\frac{A}{4 l_{\mathrm{P}}^{2}}+\frac{1}{2} \ln \left(\frac{A}{4 l_{\mathrm{P}}^{2}}\right)+\ln \left(\frac{1}{e \sqrt{\pi}}\right) .
$$


It is the same, apart from the logarithmic corrections, as the BekensteinHawking entropy $S_{\mathrm{BH}}=A / 4 l_{\mathrm{P}}^{2}$.

Let us now consider a Kerr-Newman black hole at a temperature $T_{\mathrm{H}}=$ $\beta^{-1}$. As is well known, in the near-horizon approximation the Euclidean sector of a Kerr-Newman black hole is similar to that of a Schwarzschild black hole. So, as in the case of a Schwarzschild black hole, one can introduce the corresponding temporal $\Theta$ and spatial $\tau$ coordinates for a Kerr-Newman black hole. I define the Hamiltonian of a Kerr-Newman black hole as that of a particle moving in the potential $U=\Omega J+\frac{1}{2} \Phi Q$,

$$
H=\frac{p^{2}}{2 M}+\Omega J+\frac{1}{2} \Phi Q
$$

where all quantities have the standard meaning. Proceeding exactly as in the derivation of $(7)$, we get the equation

$$
\frac{\partial \rho}{\partial \Theta}=D \frac{\partial^{2} \rho}{\partial \tau^{2}}-b \rho
$$

where $D=\hbar / 2 k M$ and

$$
b=\frac{1}{k}\left(\Omega J+\frac{1}{2} \Phi Q\right) .
$$

This is also a diffusion equation but with the convection term $-b \rho$. The equation describes the probability flow in Euclidean sector of a Kerr-Newman black hole with both diffusion $D \partial^{2} \rho / \partial \tau^{2}$ along the axis $\tau$ and the outflow $-b \rho$ in a direction perpendicular to the axis. How much diffusion and convection takes place depends on the relative size of the two coefficients $D$ and $b$. The solution to the equation (at the initial condition (9)) is

$$
\rho=\frac{1}{\sqrt{4 \pi D \Theta}} \exp \left[-\frac{\left(\tau-\tau^{\prime}\right)^{2}}{4 D \Theta}\right] \exp (-b \Theta) .
$$

There is also an integral formula for solutions to (15), known as the FeynmanKac formula; it is an integral over path space with respect to Wiener measure [2]. Since, as in the Schwarzschild case, $\tau \sim \tau+\beta$, where $\beta=2 \pi / k$, the trace of (17) leads to

$$
Z(\beta)=\frac{\beta}{\sqrt{4 \pi D \Theta}} \exp \left(-\frac{\beta^{2}}{4 D \Theta}\right) \exp (-b \Theta) .
$$


This this the partition function for a Kerr-Newman black hole. Differentiating with respect to the $\beta$ and then putting $\Theta=2 \pi$, we obtain the internal energy

$$
E=-\frac{\partial \ln Z_{g}(\beta)}{\partial \beta}=M-\beta^{-1}
$$

It is the same, apart from the Hawking temperature, as the mass of the black hole. Finally, the entropy of a Kerr-Newman black hole is given by

$$
\begin{aligned}
S= & \ln Z+\beta E=\frac{A}{4 l_{\mathrm{P}}^{2}}+\frac{1}{2} \ln \left(\frac{A}{4 l_{\mathrm{P}}^{2}}\right)+ \\
& +\frac{1}{2} \ln \left(\frac{M}{\sqrt{M^{2}-a^{2}-Q^{2}}}\right)+\ln \left(\frac{1}{e \sqrt{\pi}}\right) .
\end{aligned}
$$

It is the same, apart from the logarithmic corrections, as the BekensteinHawking entropy of the black hole.

The statistical interpretation of the Bekenstein-Hawking entropy remains a central problem in black hole physics. The path integral approach in Euclidean quantum gravity cannot answer what the degrees of freedom are responsible for the entropy. In this note, the black hole motion in Euclidean sector is modeled as an one-dimensional diffusion in the coordinates $\tau$ and $\Theta$. A random walk process is the basis of the diffusion. Thus, if the model is correct, then there should exist the black hole constituents with the Boolean degrees of freedom $\pm \Theta$ or $\pm \tau$.

\section{References}

[1] K. Ropotenko, Phys. Rev. D 85, 104032 (2012).

[2] R.P. Feynman, Statistical Mechanics, (Benjamin, New York, 1972); M.Toda, R. Kubo, N. Saito, Statistical Physics I: Equilibrium Statistical Mechanics, (Springer, Berlin, 1983).

[3] G.W. Gibbons and S.W. Hawking, Phys. Rev. D 15, 2752 (1977). 